\title{
About the Choice of Type and Design \\ of the Passive Radio Reflector of the Complex \\ of the Passive Radar-Location Realizing \\ Uglomerno-Raznostno-Dalnomernyy the Method
}

\author{
Aleksey N. Fomin , Vitalii V. Loy*, \\ Marat R. Karimullin ${ }^{\mathrm{b}}$ and Irina I. Gorbachova ${ }^{\mathrm{c}}$ \\ ${ }^{a}$ Siberian Federal University \\ 79 Svobodny, Krasnoyarsk, 660041, Russia \\ ${ }^{b}$ Military Academy of Aero-Space Defense \\ named after the Marshal of Soviet Union G.K. Zhukov \\ 50 Zhigareva Str., Tver, 170022, Russia \\ ${ }^{c}$ Krasnoyarsk Branch of the St. Petersburg University \\ of Civil Aviation \\ 15 Vzletnaya Str., Krasnoyarsk, 660135, Russia
}

Received 14.06.2018, received in revised form 14.09.2018, accepted 29.10.2018

In article the technique of the solution of a problem of the choice of type, a design and an installation site of KMP (the passive radio reflector) for the complex of a passive location realizing a goniometric and differential and ranging method is considered.

Keywords: Goniometric and differential and ranging method, a goniometric and differential and ranging method with one base, director of active hindrances, FATHERS, complex of a passive location, Communist Party of Luxembourg, PAP-KPM-RLS, the digital phased antenna lattice, TsFAR, EPR, angular radio reflector, $U R O$.

Citation: Fomin A.N., Loy V.V., Karimullin M.R., Gorbachova I.I. About the choice of type and design of the passive radio reflector of the complex of the passive radar-location realizing uglomerno-raznostno-dalnomernyy the method, J. Sib. Fed. Univ. Eng. technol., 2018, 11(7), 783-788. DOI: 10.17516/1999-494X-0104.

(c) Siberian Federal University. All rights reserved

This work is licensed under a Creative Commons Attribution-NonCommercial 4.0 International License (CC BY-NC 4.0).

* Corresponding author E-mail address: 1v2009.1974@mail.ru 


\title{
О выборе типа и конструкции
}

\section{пассивного радиоотражателя комплекса \\ пассивной радиолокации, реализующего \\ угломерно-разностно-дальномерный метод}

\author{
А.Н. Фомин ${ }^{\text {a }, ~ В . В . ~ Л о и ̆ а ~}$ \\ М.Р. Каримуллин ${ }^{\tilde{0}}$, И.И. Горбачева ${ }^{\mathrm{B}}$ \\ ${ }^{a}$ Сибирский федеральный университет \\ Россия, 660041, Красноярск, пр. Свободныий, 79 \\ ${ }^{6}$ Военная академия воздушно-космической обороны \\ им. Маршала Советского Союза Г.К. Жукова \\ Россия, 170022, Тверь, ул. Жигарева, 50 \\ ${ }^{6}$ Красноярский филиал Санкт-Петербургского университета \\ гражданской авиации \\ Россия, 660135, Красноярск, ул. Взлетная, 15
}

В статье рассматривается методика решения задачи выбора типа, конструкции и места установки КМП (пассивного радиоотражателя) для комплекса пассивной локаџии, реализующего угломерно-разностно-дальномерный метод.

Ключевые слова: угломерно-разностно-дальномерный метод, постановщик активных помех, ПАП, комплекс пассивной локачии, КПЛ, ПАП-КПМ-РЛС, иифровая фазированная антенная решетка, ЦФАР, ЭПР, уголковый радиоотражатель, УРО.

В настоящее время в системе ПВО основным средством воздушной разведки выступают активные радиолокационные средства. Это предъявляет высокие требования к ним и вызывает дополнительные затраты ресурсов на совершенствование их систем борьбы с активными помехами, что не всегда оправданно при низкой живучести и высокой стоимости данных РЛС. Несмотря на принимаемые меры по обеспечению в активных РЛС большой дальности обнаружения и определения координат средств воздушного нападения (CBH), в силу объективных законов физики, всегда энергетически и экономически эффективнее ставить помехи, чем создавать системы защиты от них.

Высокий уровень развития теории пассивной локации позволяет осуществлять обнаружение воздушных объектов по их собственному излучению на дальностях, при которых мощность принятого сигнала может явно не выделяться над уровнем внутренних шумов приемного тракта [1].

При этом реализация методов обнаружения воздушных объектов в комплексах пассивной локации (КПЛ) дает возможность обеспечить высокую скрытность и живучесть радиотехнических систем. Однако отсутствие в таких комплексах возможности обнаруживать цели, осуществляющие полет в режиме радиомолчания, не позволяет в полной мере полагаться на их информацию при организации системы ПВО. Для устранения основного недостатка КПЛ, ограничивающего их широкое применение, в работе $[2,3]$ проводится исследование возможности использования сигналов от постановщиков активных помех 


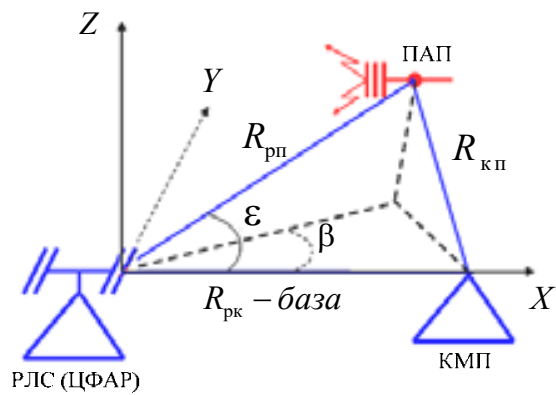

Рис. 1. Геометрия определения координат ПАП угломерно-разностно-дальномерным методом с одной базой

Fig. 1. Geometry determine the coordinates PAP goniometric-differential-distance measuring method with a single base

(ПАП) и других источников излучения с помощью угломерно-разностно-дальномерного метода.

Это связано с тем, что данный метод энергетически наиболее выгоден среди всех существующих методов пассивной локации за счет использования высоконаправленной антенны с большим коэффициентом усиления и при использовании электромагнитной энергии одного ПАП позволяет однозначно оценить координаты любого количества объектов, так как система уравнений имеет единственное решение.

Реализация данного метода предполагает использование цифровой фазированной антенной решетки (ЦФАР), расположенной в основном пункте приема. ЦФАР формирует две ДНА: первая образует целевой канал (ЦК) в направлении на воздушный объект (ВО) или контрольный местный предмет (КМП), вторая - опорный канал (ОК) в направлении на ПАП. При этом под КМП понимается возвышенность искусственного или естественного происхождения, находящаяся в зоне прямой видимости РЛС и имеющая точно известные координаты, при этом положение энергетических центров «блестящих точек» позволяет однозначно оценить координаты этого КМП. В соответствии с положениями угломерно-разностно-дальномерного метода пассивной локации на рис. 1 поясняется геометрия определения координат ПАП с одной базой.

На рис. 1 показана РЛС, которая формирует две достаточно узкие ДНА - на ПАП и КМП. На ПАП сформирован ОК для приема эталонного сигнала для корреляционного обнаружителя. На КМП формируется ЦК для приема отраженного сигнала. По сигналу, принятому непосредственно от ПАП, и сигналу, отраженному от КМП, с помощью корреляционного обнаружителя определяется разность расстояний, пройденных сигналами, принятыми напрямую от ПАП и переотраженного от КМП.

В соответствии с расположением объектов ПАП, КМП и РЛС разность расстояний, пройденных сигналами, определяется как

$$
\Delta r=\left(R_{P K}+R_{\mathrm{K \Pi}}\right)-R_{\mathrm{P \Pi}},
$$

где $R_{K П}$ - расстояние, пройденное сигналом от ПАП до КМП; $R_{P П}$ - расстояние, пройденное сигналом от ПАП до РЛС. 
Таким образом, местоположение ВО в пространстве соответствует точке пересечения двух плоскостей и поверхности гиперболоида.

$$
R_{\mathrm{P} \Pi}=\frac{R_{P K}^{2}-\Delta r^{2}}{2 \cdot\left(R_{\mathrm{PK}} \cdot \cos (\varepsilon) \cdot \cos (\beta)-\Delta r\right)},
$$

где углы $\beta$ и $\varepsilon$ характеризуют азимут и угол места ПАП относительно линии базы; $R_{P K}-$ база системы (Б), расстояние от точки стояния РЛС до КМП; $\Delta r$ - разность расстояний, пройденных сигналами от ПАП до РЛС и переотраженного сигнала от КМП до РЛС, которая определяется согласно выражению (1).

Таким образом, по переотраженной электромагнитной энергии ПАП от КМП определяется местоположение самого ПАП, которое оценивается по угловому положению ДНА ( $\beta$ и $\varepsilon)$ целевого канала (ЦК) и наклонной дальности до ПАП $R_{P \Pi}(3)$.

Важной и актуальной задачей при реализации данного метода определения местоположения ПАП является выбор типа, конструкции и места установки КМП (пассивного отражателя). Методика решения подобной аналогичной задачи была предложена и исследована в работе [4].

В частности, в качестве пассивного радиоотражателя как энергетически, так и экономически целесообразно использовать уголковый радиоотражатель (УРО), дающий хорошее отражение в широком диапазоне углов падения приходящего радиосигнала. ЭПР треугольного УРО зависит от длины его ребра и длины волны и рассчитывается по формуле

$$
\sigma_{\mathrm{yo}}=\frac{4 \pi \cdot a^{4}}{3 \cdot \lambda^{2}}
$$

где $a$ - длина ребра; $\lambda$ - длина волны.

Для увеличения ЭПР несколько УРО объединяют в один конструктивный модуль. Варианты компоновки модуля УРО для КМП будут рассмотрены ниже. Чтобы произвести размеры УРО и его конфигурацию, необходимо произвести расчет радиолинии ПАП-КПМ-РЛС, для чего воспользуемся методикой, изложенной в работе [4]. Задавши в качестве первого приближения вероятность правильного обнаружения, равную 0,9 , и вероятность ложной тревоги $10^{-6}$ при отношении сигнал/шум, равную 10 , можно установить максимальную дальность определения местоположения ПАП в соответствии с уравнением радиосвязи для разнесенных приемных и передающих пунктов как

$$
R_{\mathrm{P} I \text { max }}=\sqrt{\frac{P_{\Pi А \Pi} \cdot G_{\Pi А \Pi} \cdot \sigma_{K M \Pi \Pi} \cdot A_{Э \Phi}}{(4 \cdot \pi)^{2} \cdot P_{\text {цmin }} \cdot R_{\mathrm{PK}}^{2}}},
$$

где $P_{\text {ПАП }}-$ спектральная плотность мощности АП; $G_{\text {ПАП }}$ - коэффициент усиления антенны ПАП;

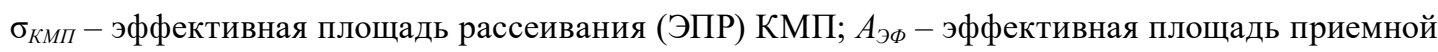
антенны РЛС; $P_{\text {u min }}$ - минимальная мощность сигнала, достаточная для обнаружения ПАП; $R_{P K}$ - база системы (Б), расстояние от точки стояния РЛС до КМП.

Согласно расчетам, приведенным в $[2,3]$ для максимальной дальности определения местоположения ПАП при длине волны $\boldsymbol{\lambda}=\mathbf{0 , 2 3} \mathbf{~ м , ~ в е л и ч и н а ~ Э П Р ~ К М П ~ д о л ж н а ~ б ы т ь ~ в ~ п р е д е л а х ~}$

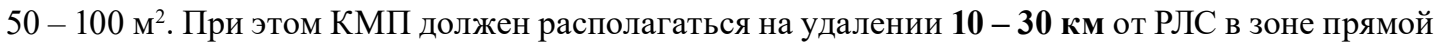
видимости. 
Выбор облика модуля уголкового радиоотражателя

Модуль УРО должен обеспечивать отражение сигнала в углах от 0 до $180^{\circ}$ (верхняя полусфера). Компоновка из четырех УРО (рис. 2) позволит отражать сигналы ПАП при всевозможных положениях модуля относительно постановщика помех. Недостатком такой компоновки является провал ЭПР при нахождении ПАП в зените над модулем УРО.

На рис. 3 представлен вариант компоновки модуля УРО из четырех треугольных УРО и одной двухгранной пластины посередине. Такая конструкция модуля УРО позволит избежать отмеченного выше недостатка.

Таким образом, описанная выше методика позволяет решить задачу выбора типа, конструкции и места установки КМП (пассивного радиоотражателя) для комплекса пассивной локации, реализующего угломерно-разностно-дальномерный метод. Для подтверждения положительных выводов предложенной методики необходимо проведение экспериментальных измерений ЭПР составных модулей УРО.

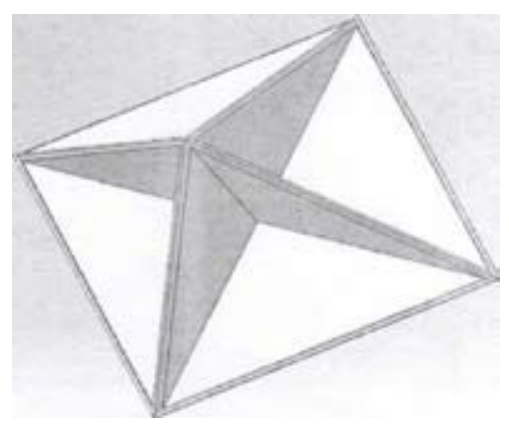

Рис. 2. Трехмерная модель модуля из четырех УРО

Fig. 2. A three-dimensional model of a module of four URO

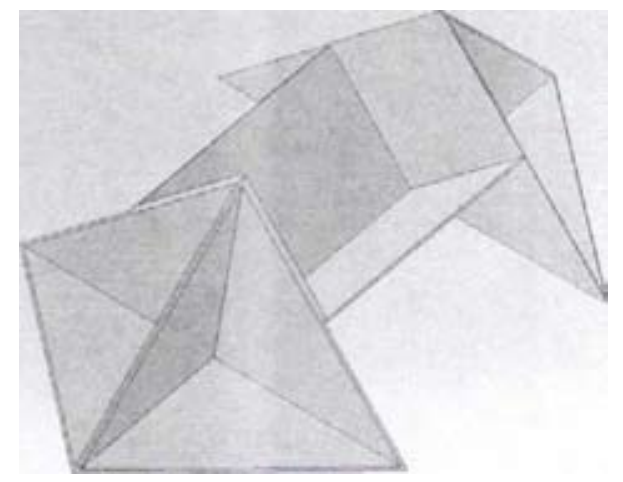

Рис. 3. Трехмерная модель модуля из четырех УРО и одной двухгранной пластины

Fig. 3. Three-dimensional model of the module of four URO and one two-sided plate 


\section{Список литература}

[1] Ширман Я.Д. Радиоэлектронные системы: основы построения и теория. Справочник. М.: ЗАО Маквис, 1998. [Shirman J.D. Electronic systems: fundamentals of construction and theory. Reference book. M.: CJSC Makvis, 1998 (in Russian)]

[2] Бердышев В.П., Каримуллин М.Р., Лой В.В. Основные этапы метода наблюдения воздушных объектов с использованием сигналов от постановщиков активных помех. Вестник военной академии воздушно-космической обороны, 2017, Тверь: ВА ВКО, 29-35. Инв.61821в. 6 с. [Berdyshev V.P., Karimullin M.R., Loii V.V. The main stages of the method of observation of air objects using signals from the Directors of active interference. Bulletin of the military Academy of aerospace defense, 2017, Tver: VA East Kazakhstan region, 29-35. Inv.61821в. 6 s. (in Russian)]

[3] Бердышев В.П., Лой В.В., Каримуллин М.Р., Цыганов А.А. Программа расчета дальности обнаружения воздушных объектов, подсвеченных источником активных помех, коррелящионно-базовой станцией пассивной локащии. Свидетельство о государственной регистрации программ для ЭВМ № 2018612425. Заявка № 2017661997, дата поступ. 21.11.17, дата регистр. 16.02.2018. М.: ФГУ ФИПС, 2018. [Berdyshev V.P., Loii V.V., Karimullin, M.R., Tsyganov A.A. Program for calculating the range of detection of air objects highlighted by the source of active interference, the correlation-base station of passive location. Certificate of state registration of computer programs No. 2018612425. Application No. 2017661997, the date of the receipt. 21.11.17, date register. 16.02.2018. M.: FGU FIPS, 2018 (in Russian)]

[4] Грицан О.Б., Бадертдинов А.М., Чеботарев В.Е., Фомин А.Н. Спутниковая радиоизмерительная система с уголковыми радиоотражателями. Сборник материалов Международной Сибирской конференции по контролю и связи (СИБКОН-2013). Раздел 2. Красноярск, 2013, 29-35 [Gritsan O.B., Badretdinov A.M., Chebotarev V.E., Fomin A.N. Satellite radio system with reflex radiotraces. Proceedings of the international Siberian conference on control and communication (SIBCON-2013). Section 2. Krasnoyarsk, 2013, 29-35 (in Russian)] 